\title{
INTERFERENCE ISSUES IN SILICON RFIC DESIGN
}

\author{
Zhaofeng Zhang, Alan Pun, Jack Lau \\ Department of Electrical and Electronic Engineering \\ The Hong Kong University of Science and Technology \\ Clear Water Bay, Kowloon, Hong Kong \\ E-mail: eezzf@ee.ust.hk Fax: (852) 23581485
}

\begin{abstract}
Traditional noise immunity methodologies used in PCB designs are less effective when applied to RF ICs. We present here analyses of both electromagnetic interferences and spiral inductor induced substrate noise in silicon RFICs that can be an impediment in achieving higher integration. In the analysis, we (1) compare the effectiveness of 4 shielding solutions in a triple layer metal technology, (2) contrast the interference on both heavily doped and lightly doped substrates, (3) study the impact of physical separation and geometrical variations, (4) and measure the inductor induced substrate noise on a 0.8 um triple-layer CMOS process.
\end{abstract}

\section{INTRODUCTION}

Three options are normally at the disposal of RF PCB layout designers to minimize interference: (a) ground shielding, (b) separation by distance, and (c) a metallic shielding box. The integration of RF components has dramatically lessened the effectiveness of these options. In this report, we discuss the effectiveness of applying the limited number of metals in an IC for interference minimization. In addition, we report on substrate noise induced through on-chip spiral inductor that is unique to RFICs.

\section{ELECTROMAGNETIC INTERFER- ENCES}

Interference in RF PCB is less problematic than that in RFICs because of two reasons: (1) There are more metal layers implemented in RF PCB. (Typical silicon-based ICs offer 3 to 5 layers of metals, while in PCB layout, double or more than double of that can be found.) Therefore, shielding in PCB is more practical. (2) Ground planes in PCBs are relatively closer to the signal layer (compared to the separation distance) and are more efficient in reducing cross talk. A simple model of crosstalk between signal lines in RF PCB at low frequencies is shown in Fig. 1a. Full wave simulation results using Sonnet[1] are shown in Fig.
$1 \mathrm{~b}$ and are compared with the analytical formula in [2]. Crosstalk will increase very sharply with the ratio of $h / d$ when $\mathrm{h} / \mathrm{d}$ is less than 0.5 . If $\mathrm{h} / \mathrm{d}$ is more than 1.0 , separation will have little advantage. For RFIC, the h/d ratio is large, as the bulk layer is in the order of 500 um thick, and minimum design rules can theoretically allow metal line separation distance of microns away.

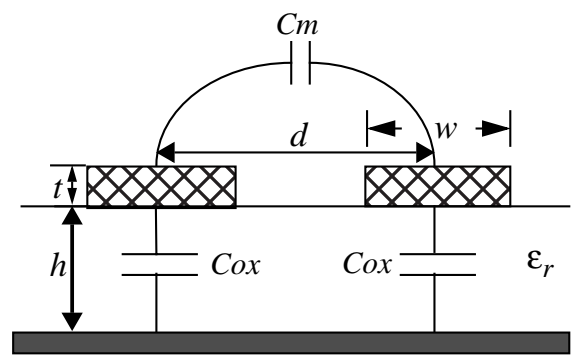

Fig. 1a. Simple model of crosstalk of RF PCB

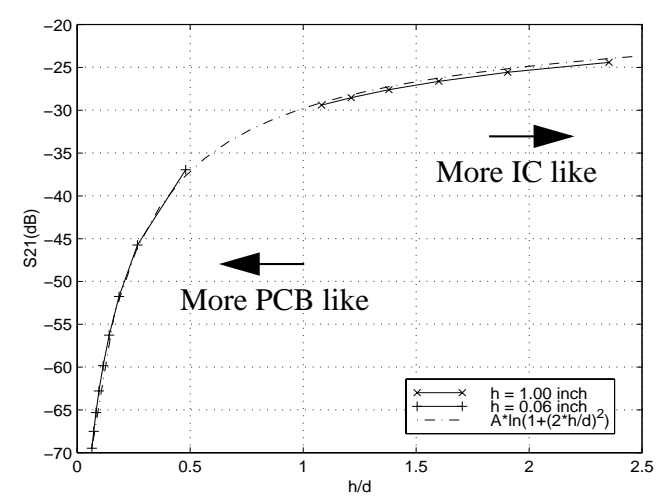

Fig. 1b. Near end crosstalk S21 of RF PCB ( $t=0.0028$ ", $w=0.025$ ", $L=0.4$ ", $\varepsilon_{r}=4.5, f=300 \mathrm{MHz}$ ).

\section{A. Simulation Setup}

Full wave electromagnetic analysis is used to study near-end and far-end crosstalk in RFICs. Both heavily doped bulk (Fig. 2a) and lightly doped bulk (Fig. 2b) are investigated. Port definition of interconnect lines are shown in Fig. 2c, and $50 \Omega$ based $S$ parameters are used in this paper. 


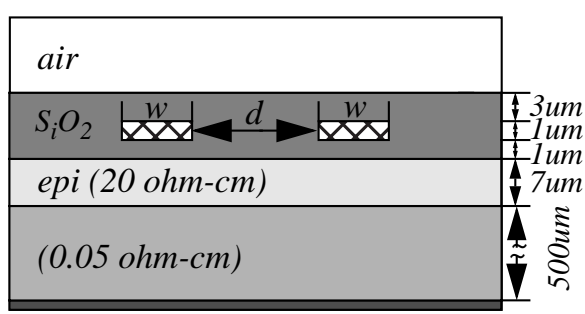

Fig. 2a. Cross section of heavily doped bulk $\left(S_{i}: \varepsilon_{r}=11.8, S_{i} O_{2}: \varepsilon_{r}=3.9, A l .: \rho=3.7 e-8 \mathrm{ohm} / \mathrm{m}\right)$.

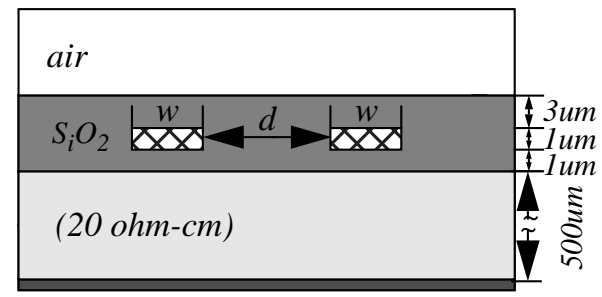

Fig. 2b. Cross section of lightly doped bulk.

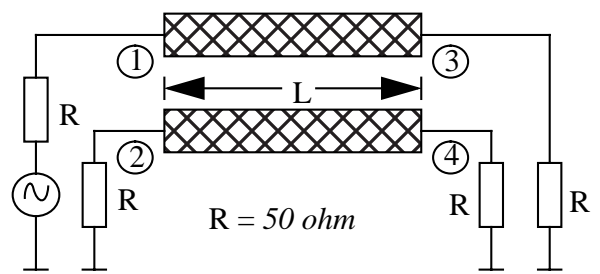

Fig. 2c. Port definition of interconnect lines

\section{B. Effect of Physical Separation (no shielding)}

Fig. 3 gives the crosstalk versus separation distance when we do not apply any shielding. Both near end crosstalk and far end crosstalk vary less than $4 \mathrm{~dB}$ within $20 \mathrm{um}$ of physical separation distance. In addition, crosstalk in lightly doped bulk is similar to that in heavily doped bulk, especially at a lower frequency band. As the frequency increases and the backside contact becomes more important, the heavily doped bulk exhibits higher noise immunity due to the more conductive bulk.

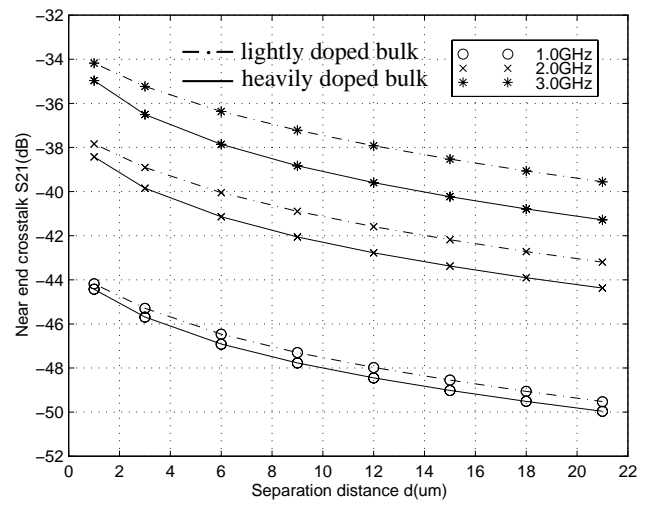

Fig. 3a. Near end Crosstalk S21 of no shielding (Fig. 2) ( w $=3$ um, $L=100 u m$ )

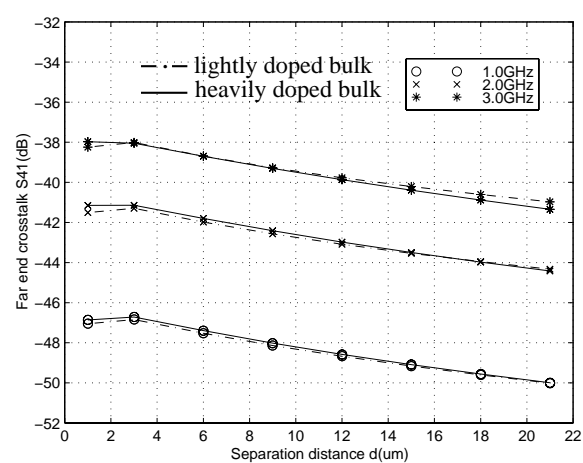

Fig. 3b. Far end crosstalk S41 of no shielding (Fig. 2) ( $w=3$ um, $L=100 u m$ )

\section{Geometries of Four Shielding Schemes}

Four possible shielding methods are proposed in this paper (Fig. 4). Ideas guiding these solutions originated from the high speed PCB layout techniques.
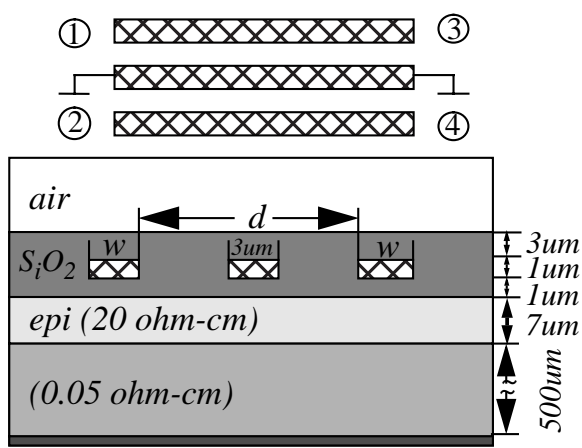

Fig. 4a. Shielding Method I.

A metal ground line is inserted in between.

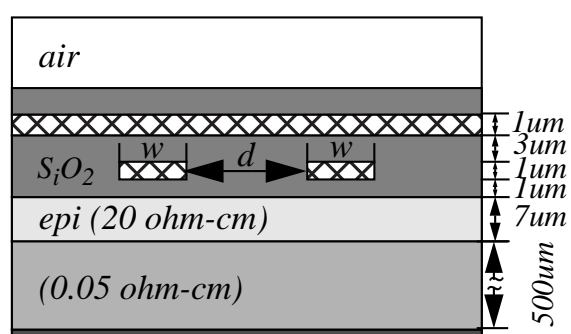

Fig. 4b. Shielding Method II

Metal 3 (top metal) is used as a ground shield.

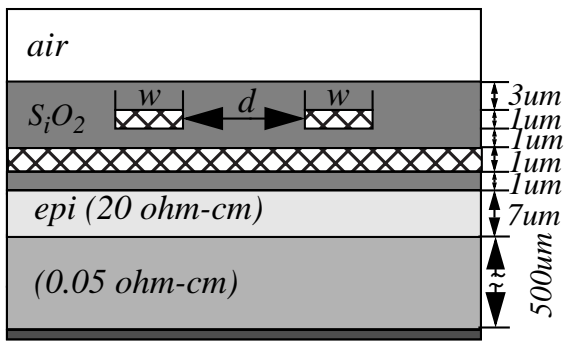

Fig. 4c. Shielding Method III.

Metal 1 (bottom layer metal) is used as a ground shield while metal 2 (middle layer metal is used for carrying signal. 


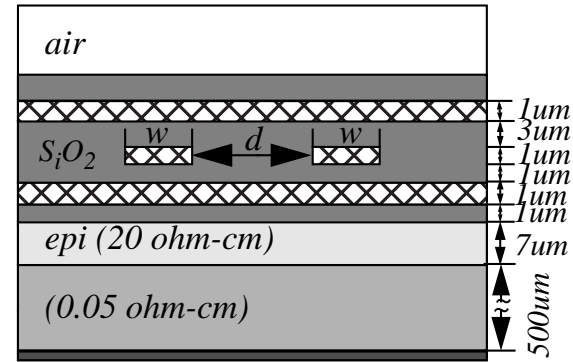

Fig. 4d. Shielding Method IV

\section{Results and Discussion}

\section{(i) Comparison between heavily doped bulk and lightly doped bulk}

As a comparison, we apply Shielding Method II (a ground plane is placed 3 um above the signal paths; Fig. $4 \mathrm{~b})$ and contrast that with the interference under no shielding (Fig 2). By placing a metal ground plane 3 um above the signal path, the coupling is reduced by $20 \mathrm{~dB}$ or more in heavily doped substrate and $10 \mathrm{~dB}$ or more in lightly doped substrate (Fig. 5). The difference in effectiveness can be attributed to the fringing field. As the line separation increases, the coupling is more dependent on the fringing field. The heavily doped substrate brings the bottom ground plane closer to the signal lines and reduces the fringing field more effectively. As the line width increases, the advantage of the heavily doped substrate is more prominent due to the larger metal to backside ground contact and more compact electrical field confinement.

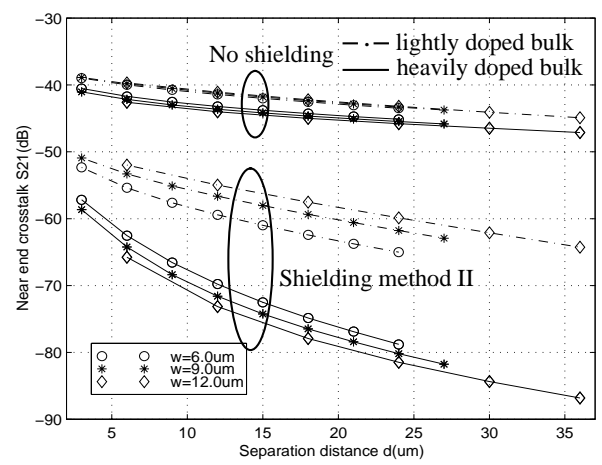

Fig. 5. Comparison between two types of bulk ( $L=100 u m, f=2.0 G H z)$

\section{(ii) Frequency properties}

Comparisons of crosstalk vs. frequency are given in Fig. 6. By placing a metal ground line in between (Shielding Method I, Fig. 4a), the coupling is reduced by $5 \mathrm{~dB}$ at $1 \mathrm{GHz}$ (Fig. 6a). Placing a large ground plane either above or below the signal lines (Fig. 4b 4c) helps reduce the coupling by another $10 \mathrm{~dB}$ (Fig. 6a). As the frequency increases, the need to eliminate the fringing field increases and the ground plane needs to be closer. At $1 \mathrm{GHz}$, the effectiveness of the ground plane being 1 um or 3 um away are roughly the same. However, at $3 \mathrm{GHz}$, a ground plane of 1 um away reduces the coupling by more than $5 \mathrm{~dB}$. The situation is the same for both near end and far end coupling (Fig. 6b)

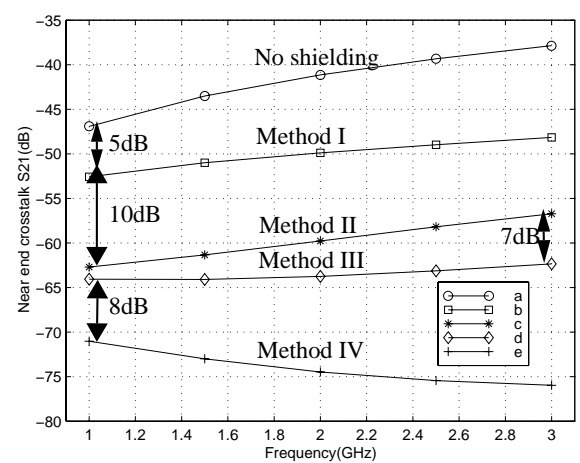

Fig. 6a. Near end crosstalk S21 vs. frequency. ( $w=3$ um, $d=9 u m, L=100 u m$ ) a: no shielding, b: method I, c: method II, d: method III, e: method IV.

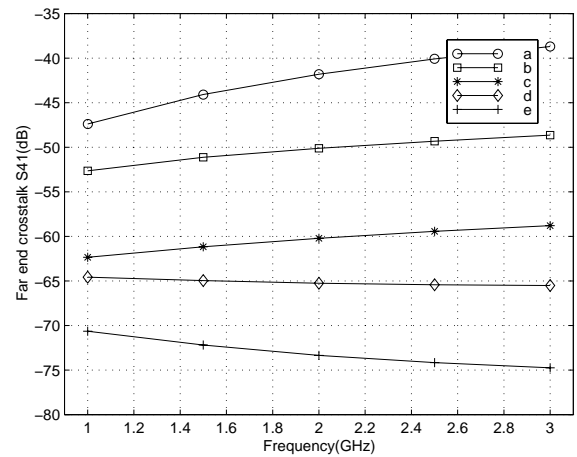

Fig. 6b. Far end crosstalk S41 vs. frequency. ( $w=3$ um, $d=9 u m, L=100 u m$ ) a: no shielding, b: method I, c: method II, d: method III, e: method IV.

\section{(iii) Effect of physical separation}

The most dramatical improvement in coupling occurs when the fringing field is completely eliminated with a ground plane both above and below the signal line (Shielding Solution IV, Fig. 4d). The coupling capacitance becomes fringe field limited as physical separation increases, approaching the $2 \mathrm{pF} / \mathrm{cm}$ limit as suggested in [3]. The near complete shielding thus has the largest impact as physical separation increases (Fig. 7). While the solution is extremely area intensive, it helps to elucidate the characteristics of the coupling here.

\section{INTEGRATED SPIRAL INDUCTOR SUBSTRATE NOISE}

Substrate noise, the kind of noise current that is injected into the substrate from active devices, has received 


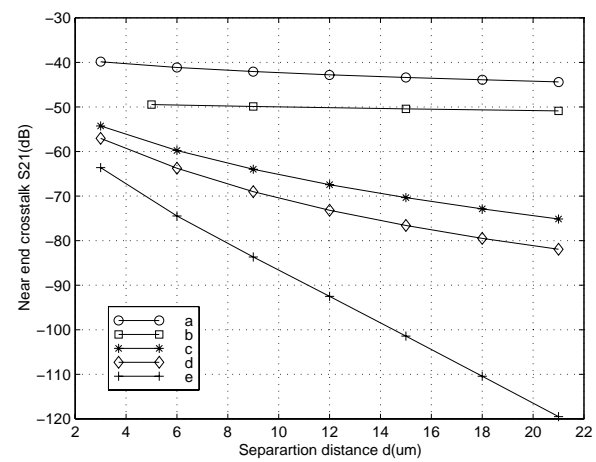

Fig. 7a. Near end crosstalk S21 vs. separation distance. ( $w=3$ um, $L=100 u m, f=2.0 \mathrm{GHz}$ ) a: no shielding, b: method I, c: method II, d: method III, e: method IV.

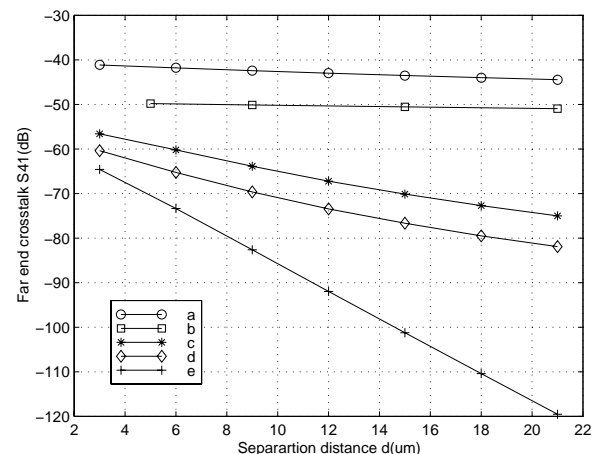

Fig. 7b. Far end crosstalk S41 vs. separation distance. ( $w=3$ um, $L=100 u m, f=2.0 \mathrm{GHz}$ ) a: no shielding, b: method I, c: method II, d: method III, e: method IV

considerable attention in mixed signal design[4-6]. We study here another mechanism that introduces substrate noise at RF through a spiral inductor. At RF, the excitement of integrated spiral inductor induces substrate noise. In an experiment, we fabricated a $2 \mathrm{nH}$ inductor in a 3 layer metal 0.8 um CMOS process on heavily doped substrate. To measure the substrate noise, we connect one end of the inductor to one port of a network anlayzer and the other port to a $\mathrm{P}^{+}$ohmic contact (Fig. 8). S21 is measured for cases when a $\mathrm{P}^{+}$guard ring is connected to the ground and floated. Coupling as high as $20 \mathrm{~dB}$ is measured. (Fig. 9). The coupling is quite insensitive to physical distance separation. The result is consistent with the prediction in [4] that the heavily doped substrate becomes an equal-potential node when the separation distance is large.

\section{CONCLUSION}

Two kinds of interferences are studied in this paper. It is shown that physical separation is pointless if no shielding schemes are adopted. Shielding obtains much better noise immunity to electromagnetic interference. $\mathrm{A} \mathrm{P}^{+}$ guard ring is very efficient in suppressing the interference

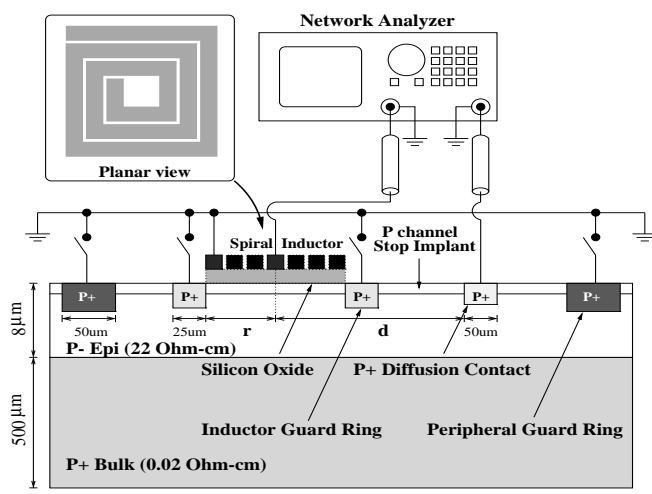

Fig. 8. Experimental setup for inductor induced substrate noise

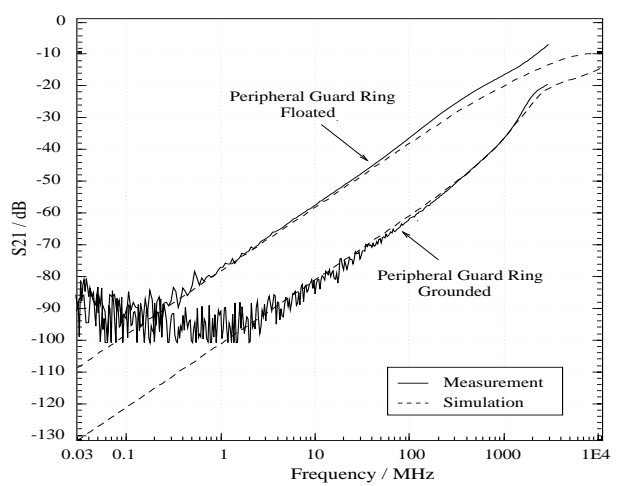

Fig. 9. Measurement and simulation of S21 vs. frequency at $\mathrm{d}=1200 \mathrm{um}$ for peripheral guard ring floated and grounded.

induced by a spiral inductor.

\section{ACKNOWLEDGEMENT}

The authors gratefully acknowledge the support of HKTIIT and RGC. Special thanks go to Mr. Jim Merrill from Sonnet Software for his help.

\section{REFERENCES}

[1] SONNET User's Manual, SONNET Software, Inc. [2] C. S. Walker, Capacitance, Inductance, and Crosstalk Analysis, pp. 62-66, Artech House, Boston, 1990.

[3] H. B. Bakoglu, Circuits, Interconnections, and Packaging for VLSI, Addison-Wesley, 1990.

[4] D. K. Su, et. al. "Experimental results and modeling techniques for substrate noise in mixed-signal integrated circuits", IEEE JSSC, pp. 420-430, Oct. 1993.

[5] K. Joardar, "Substrate crosstalk in BiCMOS mixed mode integrated circuits", IEEE JSSC, vol. 29, no. 10, pp. 1212-1219, Oct. 1994,

[6] T. Blalack, J. Lau, F. J. R. Clement, and B. A. Wooley, "Experimental results and modeling of noise coupling in a lightly doped substrate", Proc. IEEE International Electron Devices Meeting, San Francisco, CA, 1996. 